\title{
Optimal number of reference points in deformation monitoring
}

\author{
H. S. Kutoglu M. Berber
}

Received: 11 September 2014/Accepted: 20 February 2015/Published online: 4 March 2015

(C) Akadémiai Kiadó 2015

\begin{abstract}
A complete deformation analysis process requires adequate consideration of all sources of errors from the initial design of the network to the parameter estimation. In this study, during deformation analysis, effect of having more object points in geodetic networks is researched. Numerical results confirm the theory that having more object points is also an important factor for deformation analysis.
\end{abstract}

Keywords Geodetic networks · Deformation monitoring · Reference points · Reliability · Redundancies - Absorption rate

\section{Introduction}

In essence, geodetic networks are established to realize geodetic coordinate systems which are defined theoretically. Besides, they are also useful to monitor surface deformations. Geodetic networks created for deformation analysis are defined as 1D, 2D and 3D networks depending on the measurement techniques used. If only leveling is run on a geodetic network, this network is considered 1D network and it may be used; for example, to observe subsidence due to mining activities or uplifting by tectonic movements. If only direction and distance measurements are made on a geodetic network, this network is considered 2D network and these type of networks among other things may be used to monitor horizontal surface deformations. By combining the aforementioned techniques 3D networks can also be formed; nonetheless, because of technical issues in practice they are

\section{H. S. Kutoglu}

Geomatics Engineering Department, Faculty of Engineering, Bulent Ecevit University, Zonguldak, Turkey

M. Berber $(\bowtie)$

Department of Civil and Geomatics Engineering, California State University, Fresno, CA, USA

e-mail: muberber@csufresno.edu 
not preferred. Using Global Navigation Satellite System (GNSS), the most popular positioning technique of our time, deformations can be observed precisely in 3D; for instance, they are used to monitor tectonic movements, to observe structural deformation such as deformations in dams, bridges etc.

Number of observations taken in a geodetic network shapes network geometry. On the other hand, the parameters which are needed to connect this network to a coordinate system are known as datum parameters. These parameters are determined by using points whose coordinates are already known in the related coordinate system. The minimum number of reference points needed for determining datum parameters of a geodetic network varies depending on the dimension of the network and the observation techniques used. Table 1 shows the reference points needed in most commonly used networks.

In deformation analysis, point coordinates must not be affected by the adjustment method. Constrained adjustment method in which reference point coordinates must remain fixed is not suitable for this approach. Hence, for the computations of coordinates of new points, called object points, which are set up to observe probable deformations, free network adjustment which does not affect the network geometry is preferred. To quantify deformations, the geodetic network is surveyed to gain multiple periods of data-minimum two periods of data is needed. In classical deformation analysis, each period of data is adjusted separately using free adjustment technique. Object points must stay stable between periodic observations. If it is found out that an object point has moved between periodic observations, this point must be removed from reference point classification. To determine if a point moved between periods, the coordinates which are obtained for each period of data are transformed using similarity transformation. By making use of this transformation, presence of outliers in the data is investigated. If an outlier point appears, this means that this point moved between periodic observations and therefore since it cannot be included into datum determination process, it must be removed from reference point classification. After making sure that reference points were stable, all observations are adjusted together using free adjustment and final coordinates of the points are determined. Finally, the coordinate differences (obtained by adjustment of periodic observations) of object points are analyzed to find out whether these differences are significant. Here, stable points mean object points serving for the determination of displacements. Numerous approaches have been suggested by different authors (Chen 1983) to determine the stability of the reference points, for instance, methods developed by Pelzer (1974), Heck et al. (1977), Lazzarini (1977), Polak (1978), van Mierlo (1978) and Niemeier (1981).

Table 1 Minimum number of reference points required in geodetic networks

\begin{tabular}{lllll}
\hline $\begin{array}{l}\text { Type of } \\
\text { network }\end{array}$ & $\begin{array}{l}\text { Observation } \\
\text { technique }\end{array}$ & $\begin{array}{l}\text { Number of } \\
\text { datum } \\
\text { parameters }\end{array}$ & $\begin{array}{l}\text { Kind of datum } \\
\text { parameters }\end{array}$ & $\begin{array}{l}\text { Minimum number } \\
\text { of reference } \\
\text { points required }\end{array}$ \\
\hline 1D networks & Leveling & 1 & 1 translation \\
2D networks & Directions & 4 & 2 translations, 1 rotation, 1 scale & 2 \\
& Distances & 3 & 2 translations, 1 rotation & 2 \\
& $\begin{array}{l}\text { Directions, distances } \\
\text { and azimuth }\end{array}$ & 2 & 2 translations & 1 \\
& Directions and distances & 3 & 2 translations, 1 rotation & 2 \\
3D networks & GPS baseline vectors & 3 & 3 translations & 1 \\
\hline
\end{tabular}


In this study, free-network adjustment of each epoch is evaluated separately, however, there are other approaches available for use. In this regard, interested readers are referred to International Study Group of FIG Commission 6 reports which are available on FIG website (fig.net). In addition, readers are referred to Chrzanowski et al. (2003) and Chrzanowski (2006) for historical development of deformation analysis. Further, Chen (1983) elucidates amply on analysis of deformation surveys.

The deficiency of the approach described above is that it is not clear whether the number of object points used is enough for reliable deformation analysis. If the number of object points used is not enough, the changes in the point coordinates that moved during the process might be absorbed by other point coordinates during the computations. In this case, during the test, which is utilized to detect the reference points which have moved, these changes cannot be determined. To prevent this during the design stage one has to work with a number reference points which are enough for a reliable deformation analysis. In this study, computation algorithms which help in this regard are outlined.

\section{Definition of datum parameters in free network adjustment}

In geodetic network adjustments the relation between the observations and unknowns are given as

$$
\mathbf{v}=\mathbf{A x}-\boldsymbol{l}
$$

where $\mathbf{A}$ is design matrix, $\mathbf{x}$ is unknown vector, $\boldsymbol{l}$ is observation vector and $\mathbf{v}$ is residual vector. Additionally, in free network adjustments, to determine datum parameters for the network, the following constraint equation is employed (Demirel (2005), Koch (1987) and Mikhail and Ackermann (1976)).

$$
\mathbf{B}^{T} \mathbf{x}=\mathbf{0}
$$

As a matter of fact, this constraint equation constitutes the coefficients of the similarity transformation which transform the network onto the reference points. In this sense, to determine the datum parameters tabulated in Table 1, using similarity transformation principles, $\mathbf{B}$ matrix is defined for $1 \mathrm{D}$ networks as

$$
\mathbf{B}^{T}=\left[\begin{array}{lllll}
1 & 1 & 1 & \ldots & 1
\end{array}\right]
$$

For 2D direction only networks as

$$
\mathbf{B}^{T}=\left[\begin{array}{rrrrrrrr}
1 & 0 & 1 & 0 & \ldots & \ldots & 1 & 0 \\
0 & 1 & 0 & 1 & \ldots & \ldots & 0 & 1 \\
-y_{1}^{0} & \boldsymbol{x}_{1}^{0} & -\boldsymbol{y}_{2}^{0} & \boldsymbol{x}_{2}^{0} & \ldots & \ldots & -\boldsymbol{y}_{p}^{0} & \boldsymbol{x}_{p}^{0} \\
\boldsymbol{x}_{1}^{0} & \boldsymbol{y}_{1}^{0} & \boldsymbol{x}_{2}^{0} & \boldsymbol{y}_{2}^{0} & \ldots & \ldots & \boldsymbol{x}_{p}^{0} & \boldsymbol{y}_{p}^{0}
\end{array}\right]
$$

For 2D direction and distance networks as

$$
\mathbf{B}^{T}=\left[\begin{array}{rrrrrrrr}
1 & 0 & 1 & 0 & \ldots & \ldots & 1 & 0 \\
0 & 1 & 0 & 1 & \ldots & \ldots & 0 & 1 \\
-\boldsymbol{y}_{1}^{0} & \boldsymbol{x}_{1}^{0} & -\boldsymbol{y}_{2}^{0} & \boldsymbol{x}_{2}^{0} & \ldots & \ldots & -\boldsymbol{y}_{p}^{0} & \boldsymbol{x}_{p}^{0}
\end{array}\right]
$$

and for 3D GNSS networks as 


$$
\mathbf{B}^{\boldsymbol{T}}=\left[\begin{array}{llllllllllll}
1 & 0 & 0 & 1 & 0 & 0 & \ldots & \ldots & \ldots & 1 & 0 & 0 \\
0 & 1 & 0 & 0 & 1 & 0 & \ldots & \ldots & \ldots & 0 & 1 & 0 \\
0 & 0 & 1 & 0 & 0 & 1 & \ldots & \ldots & \ldots & 0 & 0 & 1
\end{array}\right]
$$

The number of columns in $\mathbf{B}$ matrix is equal to the number of datum parameters and the number of rows is equal to the number of coordinates of points in the network. The rows which correspond to object points are made all zeros. As such, only a transformation onto reference points coordinates is realized. If the rows which correspond to object points are not made all zeros, this matrix is termed as $\mathbf{G}$ matrix.

Using Eqs. (1) and (2), the unknowns are determined using Least Squares method. For Least Squares solution we write

$$
\mathbf{v}^{T} \mathbf{P v}=\min
$$

and

$$
\mathbf{x}^{T} \mathbf{x}=\min
$$

By applying Eq. (8) to Eq. (2) we get

$$
\mathbf{t}=\left(\mathbf{B}^{T} \mathbf{B}\right)^{-1} \mathbf{B}^{T} \mathbf{x}
$$

This equation helps determining datum parameters which minimizes the amount of corrections which are made to coordinates of points identified as the reference points.

If enough number of reference points are not used to allow for reliable external parameter computations, potential displacements in some of these reference points coordinates may be absorbed substantially by these external parameters. If this is the case, in the second stage in which stable reference point analysis is performed, these displacements might not be unveiled. To prevent this, enough number of reference points and/or good geometry is needed, which will allow reliable external parameter computations. In order to determine adequate reference point number and geometry, reliability analysis (see the following section) for transformation parameters needs to be consulted.

Although main focus of this paper is showing the effect of having more reference points, this does not mean that design of the reference points is ignored. It is well known that the effects of errors are reflected to their corresponding residuals through their redundancy numbers. That is why the redundancy number of the observations is desired to be as high as possible. To have high redundancy number for the observations, the design (geometry) of the network is important as well as the number of redundant measurements in the network.

Redundant measurements also enable use of least squares and global test afterward. The global test is used to determine whether the adjustment as a whole is satisfactory. If the global test fails, one of the reasons might be the outliers in the data. In this case, statistical tests such as Baarda's test, Tau $(\tau)$ test is conducted see Teunissen (2000). These tests are developed on the assumption that observations are uncorrelated. Nevertheless, correlated observations are very often encountered i.e., simultaneously observed baseline vectors in GPS campaigns are correlated. For correlated observations see Wang and Chen (1994) and Schaffrin (1997).

A mathematically equivalent alternative to statistical testing is the concept of confidence regions. Confidence regions can also be determined by eigenvalues and eigenvectors since the lengths of confidence region axes are proportional to the square roots of the 
eigenvalues of covariance matrix of the estimated parameters and since the directions are given by the eigenvectors.

\section{Reliability analysis}

Reliability of observations for problems such as in Eq. (9) is given as

$$
r_{i}=\left(\mathbf{I}-\mathbf{B}\left(\mathbf{B}^{\mathrm{T}} \mathbf{B}\right)^{-1} \mathbf{B}^{\mathrm{T}}\right)_{i i}
$$

where $\mathbf{I}$ is identity matrix. As well known, in a datum transformation, observations are reference point coordinates. By making use of reliability values calculated using Eq. (10), the amount of possible error in a residual which will be applied to a reference point coordinate (in our case temporal displacements in coordinates) is estimated as

$$
\nabla_{\mathrm{i}}=\frac{\nabla \mathbf{v}_{\mathrm{i}}}{\mathrm{r}_{\mathrm{i}}} \approx \frac{\mathbf{v}_{\mathrm{i}}}{\mathrm{r}_{\mathrm{i}}}
$$

Statistically this can be calculated as

$$
\left|\nabla_{0 \mathrm{i}}\right| \geq \frac{\delta_{0}}{\sqrt{\mathrm{r}_{\mathrm{i}}}} \mathrm{m}_{\mathrm{i}},
$$

where the limit value $\nabla_{0 \mathrm{i}}$ is called the minimum detectable error, $\delta_{0}$ is the non-centrality parameter of normal distribution determined by the probability levels $\alpha$ and $\beta$ of accepting and rejecting the non-central normal distribution for the residual $i$ and $\sigma$ is the standard deviation (Leick 1995). As can be seen, as reliability value increases, the amount of error that might be detected in reference points increases with the square of the minimum detectable error.

If the error is smaller than the minimum detectable error, it cannot be detected through the outlier tests, and thus cannot be removed from the adjustment. In this case, the undetectable error is absorbed by the adjustment. The amount of the absorption caused to falsification in the adjustment is computed by the difference between the error and the impact:

$$
\mathrm{A}_{\mathrm{i}}=\nabla_{\mathrm{i}}-\nabla \mathrm{v}_{\mathrm{i}}=\frac{1-\mathrm{r}_{\mathrm{i}}}{\mathrm{r}_{\mathrm{i}}} \mathrm{v}_{\mathrm{i}}
$$

It is obvious with this equation that the absorption becomes larger than the residual with the value of $r_{i}<0.5$ (Kutoglu and Ayan 2006).

\subsection{Optimal reference point number in leveling networks}

If $\mathbf{B}$ matrix given in Eq. (3) is substituted in Eq. (10), reliability value for any reference point is calculated as

$$
\mathrm{r}_{\mathrm{H}}=1-\frac{1}{n}
$$

As can be seen with this equation reliability in leveling networks depends on only the number of reference points $n$. As a result, as the number of reference point increases, the amount of error that might be detected in reference points increase and in addition the 
Leveling Network

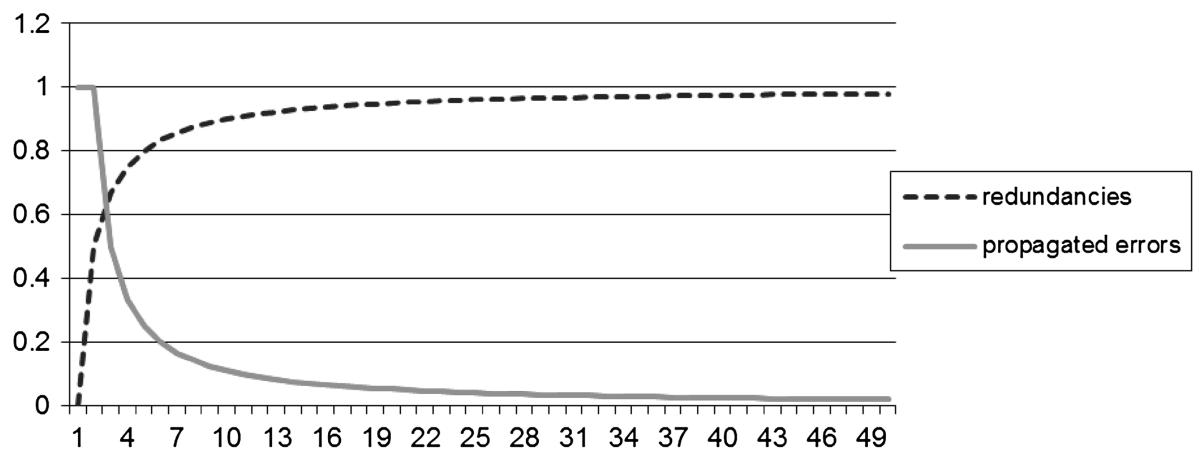

Fig. 1 Theoretical absorption rate for a leveling network (errors are given in dm unit). Horizontal axis represents the number of points and vertical axis shows the amount of error

likelihood of absorption of this error by other point coordinates decreases. To demonstrate this theory, Fig. 1 is prepared. As can be seen, as n value in Eq. (14) increases, the amount of absorption rate decreases.

To be able to show the entire picture, in Fig. 1, the number of contributing point (n) is increased up to 50 and the propagation of $10 \mathrm{~cm}$ error is shown as an example.

\subsection{Optimal reference point number in 2D networks}

The general case for 2D networks is when directions are observed. In this case, the number of datum parameters need to be determined is four and $\mathbf{B}$ matrix is formed as seen in Eq. (5). Thus, reliability values for reference point coordinates are computed as (Kutoglu 2001).

$$
\begin{aligned}
\mathrm{r}_{\mathrm{x}_{\mathrm{i}}}=\mathrm{r}_{\mathrm{y}_{\mathrm{i}}}=1-( & \frac{1}{\mathrm{n}}+\frac{[\mathrm{x}]^{2}+[\mathrm{y}]^{2}}{\mathrm{n}^{2}\left[\mathrm{x}^{2}+\mathrm{y}^{2}\right]-\mathrm{n}[\mathrm{x}]^{2}-\mathrm{n}[\mathrm{y}]^{2}}+\frac{\mathrm{x}_{\mathrm{i}}^{2}+\mathrm{y}_{\mathrm{i}}^{2}}{\left[\mathrm{x}^{2}+\mathrm{y}^{2}\right]-\left(\frac{[\mathrm{x}]^{2}+[\mathrm{y}]^{2}}{\mathrm{n}}\right)} \\
& \left.-\frac{2\left([\mathrm{x}] \mathrm{x}_{\mathrm{i}}+[\mathrm{y}] \mathrm{y}_{\mathrm{i}}\right)}{\mathrm{n}\left[\mathrm{x}^{2}+\mathrm{y}^{2}\right]-[\mathrm{x}]^{2}-[\mathrm{y}]^{2}}\right)
\end{aligned}
$$

As can be seen with this equation, unlike leveling networks, in addition to reference point number, coordinates of the points are also important.

In Fig. 2, the number of contributing point $(n)$ is increased up to 50 and the propagation of $10 \mathrm{~cm}$ error is shown as an example.

\subsection{Optimal reference point number in 3D GNSS networks}

In 3D GNSS networks, B matrix is formed as can be seen in Eq. (6). Similar to leveling networks, reliability values for any reference point coordinates which determine the datum for the network are calculated as

$$
\mathrm{r}_{\mathrm{X}_{\mathrm{i}}}=\mathrm{r}_{\mathrm{Y}_{\mathrm{i}}}=\mathrm{r}_{\mathrm{Z}_{\mathrm{i}}}=1-\frac{1}{n}
$$




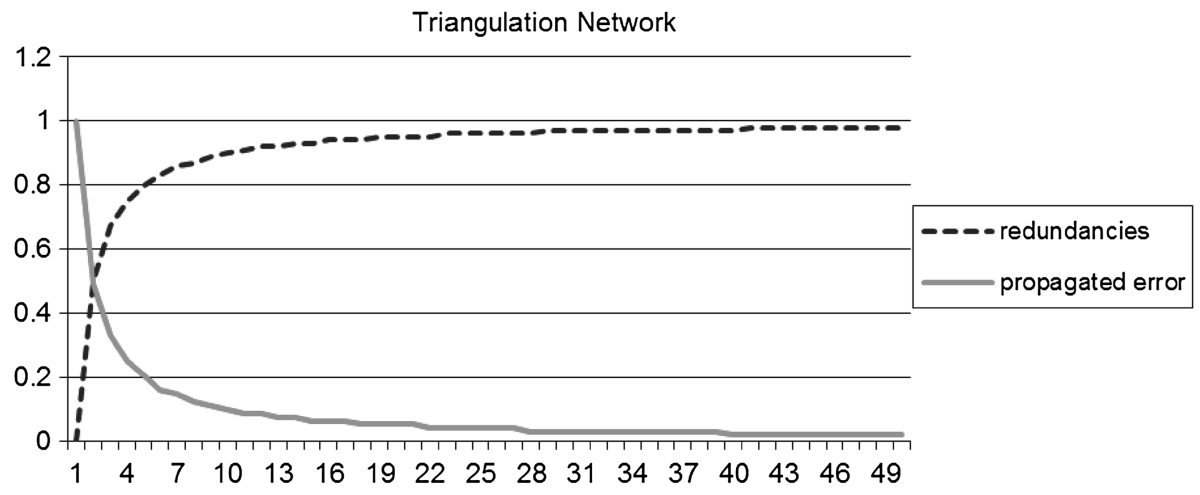

Fig. 2 Theoretical absorption rate for a triangulation network (errors are given in dm unit). Horizontal axis represents the number of points and vertical axis shows the amount of error

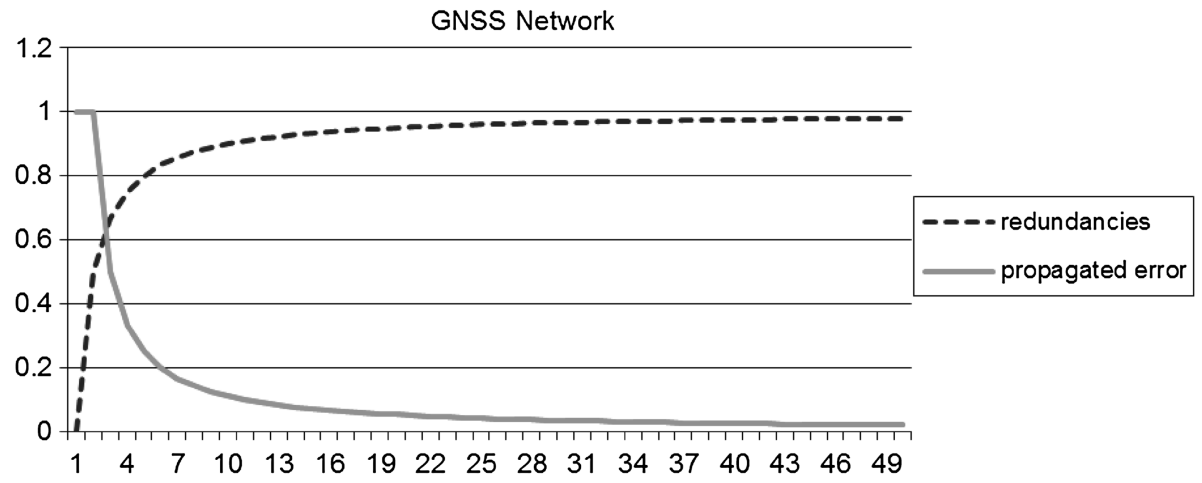

Fig. 3 Theoretical absorption rate for a GNSS network (errors are given in dm unit). Horizontal axis represents the number of points and vertical axis shows the amount of error

It means that in GNSS networks, again, the number of reference points is the only parameter for reliable datum determination. As $n$ value in Eq. (16) increases, the amount of absorption rate decreases.

In Fig. 3, contribution of 50 points and the propagation of $10 \mathrm{~cm}$ error are displayed as an example.

\section{Numerical results}

For GNSS measurements FAUNet seen in Fig. 4 is used. Measurements are made using static method and $1 \mathrm{~h}$ data is collected at each site with $5 \mathrm{~s}$ sampling rate. A triple frequency GNSS system (Leica GX1230) is used to take the measurements. This network is comprised of five object points and eight baseline observations. The network is adjusted utilizing free network adjustment outlined in Sect. 2.

As mentioned in Sect. 3, to determine optimal point number, first $10 \mathrm{~cm}$ error is given to the $3 \mathrm{D}$ coordinates of point 5 and how these errors are propagated to the coordinates of 


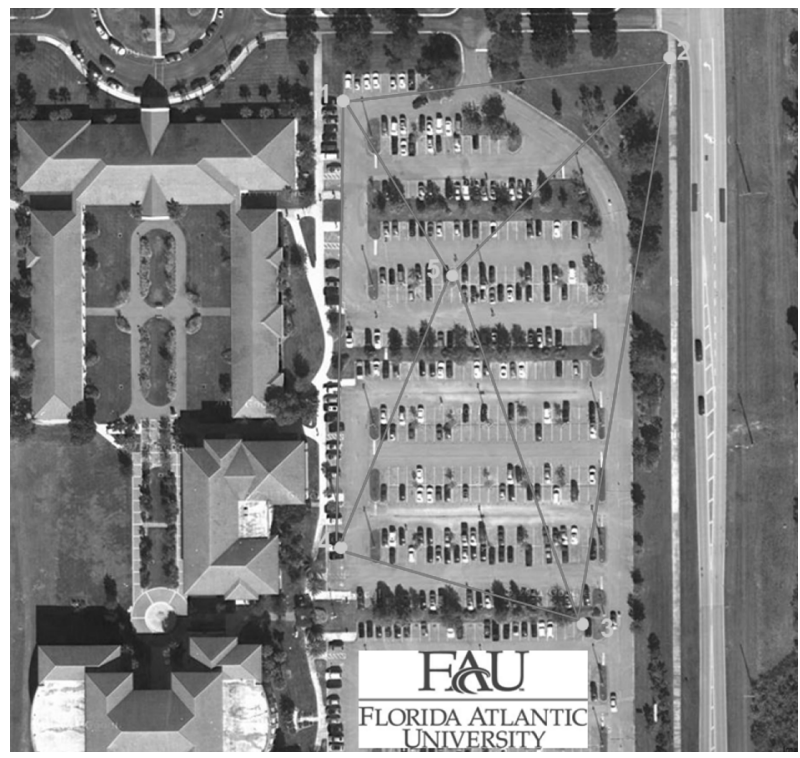

Fig. 4 FAUNet (image from Google)

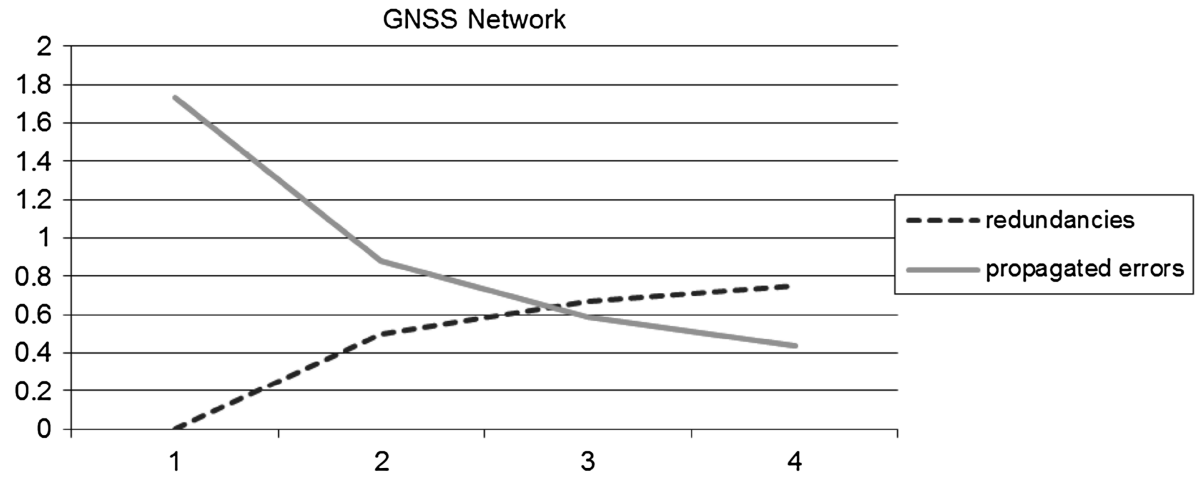

Fig. 5 Redundancies versus propagated errors in GNSS network (errors are given in dm unit). Horizontal axis represents the number of points and vertical axis shows the amount of error

other points are observed. And then, first, only the contribution of point 5 is sought by filling only the rows and columns of this point in Eq. (6). Next, the contributions of points 1, 2 and 3 are investigated respectively. Each time the amount of error propagated to the other network points are calculated by subtracting remaining error in the coordinates of this point from the amount of total given error. In Fig. 5, redundancies and propagated errors are shown for this network. It is clear with Fig. 5 that as $n$ value increases in Eq. (16), the amount of propagated error decreases.

Later on the same network seen in Fig. 4 is surveyed using Leica NA730 level and eight height difference observations are taken. Again, it is adjusted utilizing free network adjustment. Following the approach described for the GNSS network, to determine optimal reference point number, first $10 \mathrm{~cm}$ error is given to the elevation of point 5 and how this 
error is propagated to the elevations of other points is observed. And then, first, only the contribution of point 5 is sought by filling only the column of this point in Eq. (3). Next, the contributions of points 1, 2 and 3 are investigated respectively. Again, each time the amount of error propagated to the other network points are calculated by subtracting remaining error in the elevation of this point from the amount of total given error $(10 \mathrm{~cm})$. In Fig. 6, redundancies and propagated errors are shown for this network. As can be seen, as $n$ value increases in Eq. (14), the amount of propagated error decreases. Also, in this network contributions of four points (5,1,2 and 3) are portrayed.

Since FAUNet seen in Fig. 4 is designed for GNSS measurements, it was not adequate for terrestrial measurements i.e., intervisibility was not possible among some of the network points. As a consequence, another network seen in Fig. 7 is used. This is also a real network surveyed utilizing Wild T2 instrument. This triangulation network also has five object points and there are fifteen direction and three distance measurements.

To determine optimal reference point number for this network, first $10 \mathrm{~cm}$ error is given to the $2 \mathrm{D}$ coordinates of point 1 and how these errors are propagated to the coordinates of other points are observed. And then, first, only the contribution of point 1 is sought by filling only the rows and columns of this point in Eq. (5). Next, the contributions of points 2, 3 and 4 are investigated respectively. Each time the amount of error propagated to the other network points are calculated by subtracting remaining error in the coordinates of this point from the amount of total given error. In Fig. 8, redundancies and propagated errors

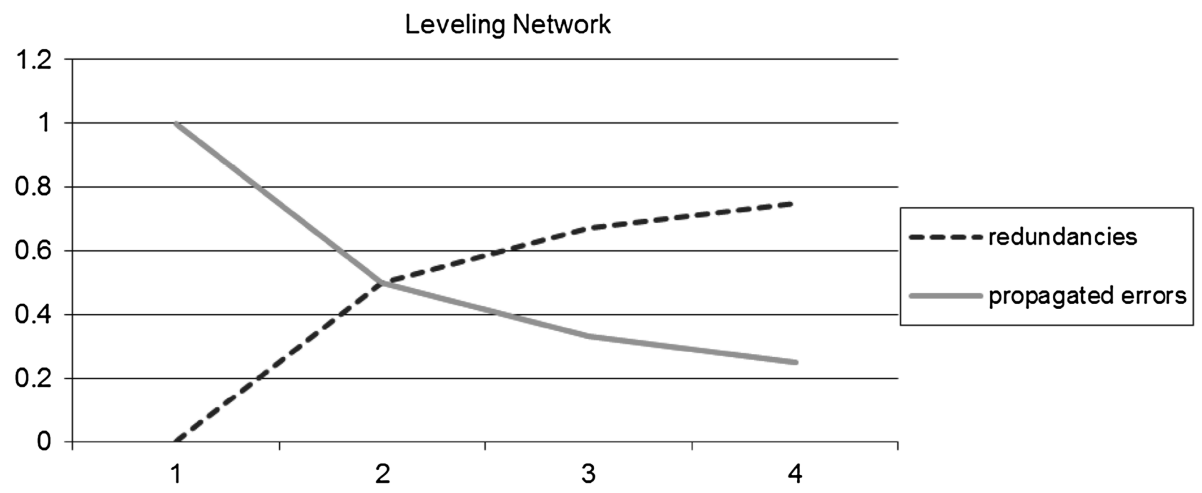

Fig. 6 Redundancies versus propagated errors in leveling network (errors are given in dm unit). Horizontal axis represents the number of points and vertical axis shows the amount of error

Fig. 7 Triangulation network

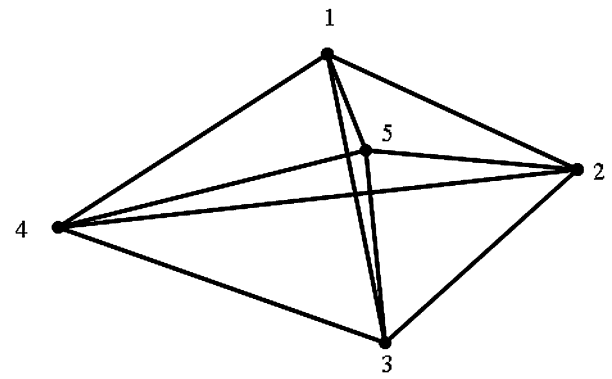




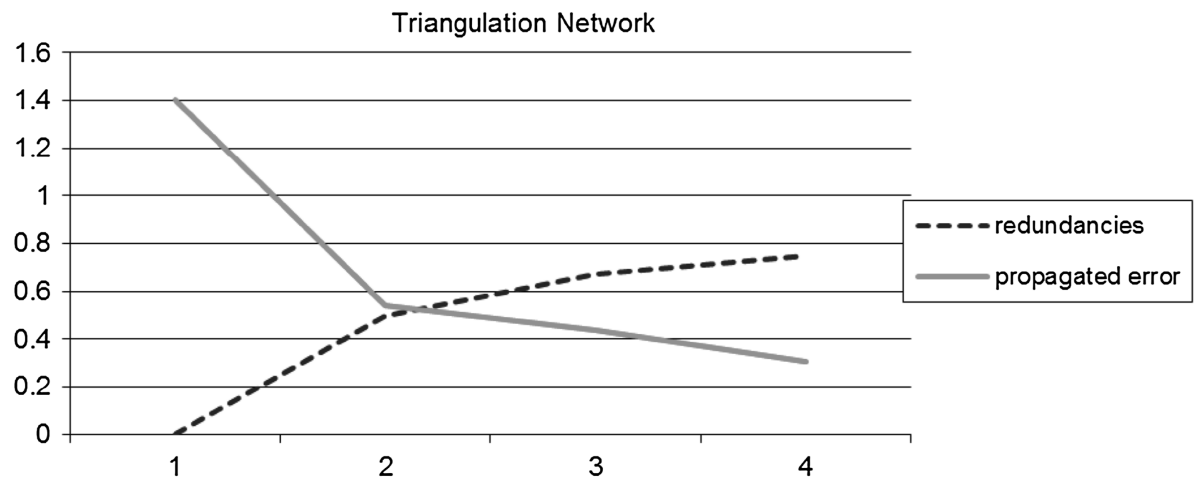

Fig. 8 Redundancies versus propagated errors in triangulation network (errors are given in dm unit). Horizontal axis represents the number of points and vertical axis shows the amount of error

are shown for this network. As can be seen in Fig. 8, as n value increases in Eq. (15), the amount of propagated error decreases.

Figures 5, 6 and 8 follow the same pattern displayed by Figs. 1, 2 and 3 and this confirms the theory.

\section{Conclusions and recommendations}

In this study, contribution of having more object points in deformation analysis is researched. It is consistent with three types of networks i.e., GNSS, leveling, triangulation that as the number of reference point increases, the error absorption rate increases and as a consequence less error is propagated to other point coordinates. This is crystal clear with GNSS and leveling networks; yet, it is not very clear with triangulation network since not only the translations but also how reference points are spread out in the project site is important. The results indicate that rather than just having reference points in solid ground for deformation analysis having more reference points is also an important factor.

Integrated monitoring schemes in which geodetic (satellite and/or terrestrial) surveys are combined with geotechnical measurements, and relatively new methods such as InSAR and laser scanning are not considered in this study; however, interested readers may develop their own approaches for these techniques using the theory presented.

\section{References}

Chen YQ (1983) Analysis of deformation surveys-a generalised method. Technical Report No. 94, Department of Surveying Engineering, University of New Brunswick, Fredericton, Canada

Chrzanowski A (2006) Tasks and Achievements of the FIG Working Group on deformation measurements and analysis. In: 3rd IAG/12th FIG symposium, Baden, 22-24 May, 2006

Chrzanowski A, Ding X, Roberts G, Whitaker C (2003) Goals and Achievements of FIG Working Group WG6.1-deformation measurements and analysis. In: Proceedings of 11th FIG symposium on deformation measurements, Santorini, Greece

Demirel H (2005) Dengeleme Hesabi. Yildiz Teknik Universitesi Basim Yayim Merkezi, Istanbul

Heck B, Kuntz E, Meier-Hirmer B (1977) Deformations-analyse mittels Relativer Fehlerellipsen. AVN $3: 78-87$ 
Koch KR (1987) Parameterschaetzung und Hypothesentests in Linearen Modellen. Dummlers Verlag, Bonn Kutoglu HS (2001) GPS Aglarinin Ulke Nirengi Aglarina Entegrasyonu. PhD Thesis, Istanbul Teknik Universitesi, Istanbul, Turkey

Kutoglu HS, Ayan T (2006) The role of common point distribution in obtaining reliable parameters for coordinate transformation. Appl Math Comput 176:751-758

Lazzarini T (1977) Determination of displacements and deformation of structures and their environment by geodetic means. In: unpublished lecture notes, "Engineering Surveys", Ed. A. Chrzanowski, Department of Surveying Engineering, University of New Brunswick, Fredericton, Canada

Leick A (1995) GPS satellite surveying, 2nd edn. Wiley, New York

Mikhail EM, Ackermann F (1976) Observations and least squares. Dun-Donnelley, New York

Niemeier W (1981) Statistical tests for detecting movements in repeatedly measured geodetic networks. Tectonophysics $71: 335-351$

Pelzer H (1974) Neuere Ergebnisse bei der statistischen Analyse von Deformationsmessungen. In: Proceedings of 14th FIG congress, Washington, D.C., paper 608-3

Polak M (1978) Examination of the stability of reference points in distance and combined angle-distance networks. In: Proceedings of the 2nd international symposium on deformation measurements by geodetic methods, Bonn, West Germany, 25-28 September, Konrad Wittwer, Stuttgart, 1981

Schaffrin B (1997) Reliability measures for correlated observations. J Surv Eng 123(3):126-137

Teunissen PJG (2000) Testing theory: an introduction. Delft University Press, The Netherlands

van Mierlo J (1978) A testing procedure for analysing geodetic deformation measurements. In: Proceedings of the 2nd international symposium on deformation measurements by geodetic methods. Bonn, West Germany, 25-28 September, Konrad Wittwer, Stuttgart, 1981

Wang J, Chen Y (1994) On the reliability measure of observations. Acta Geodaet Cartograph Sin, English Edition, pp 42-51 